\title{
Convergence property of IDR variant methods in the integral equation analysis of electromagnetic scattering problems
}

\author{
Hidetoshi Chiba ${ }^{\text {a)}}$, Toru Fukasawa, and Hiroaki Miyashita \\ Information Technology R\&D Center, Mitsubishi Electric Corporation, \\ 5-1-1 Ofuna, Kamakura, Kanagawa, Japan \\ a)Chiba.Hidetoshi@eb.MitsubishiElectric.co.jp
}

\begin{abstract}
In this study, we investigate the performance of the variants of the induced dimension reduction (IDR) in large-scale electromagnetic scattering problems. We also focus on the tolerance to the so-call spurious convergence and convergence property of the IDR variant methods. Comparative numerical experiments, using IDR(s), Bi-IDR(s), and $\operatorname{GIDR}(\mathrm{s}, \mathrm{L})$, reveal that $\operatorname{GIDR}(\mathrm{s}, \mathrm{L})$ with $\mathrm{L}=2$ or 4 and an $\mathrm{s}$ value of around 15 shows the best performance with respect to the balance between the convergence property and the tolerance to the spurious convergence for problems with several levels of geometry complexity.
\end{abstract}

Keywords: IDR variants, method of moments, multilevel fast multipole algorithm

Classification: Electromagnetic theory

\section{References}

[1] P. Sonneveld and M. B. van Gijzen: SIAM J. Sci. Comput. 31 (2009) 1035. DOI: $10.1137 / 070685804$

[2] P. Sonneveld and M. B. van Gijzen: Dept. of Applied Math. Anal., TR08-21, Delft University of Technology (2008).

[3] K. Morita, Y. Onoue and S. Fujino: $2^{\text {nd }}$ International Kyoto-Forum on Krylov Subspace Method (2010) 97.

[4] G. L. G. Sleijpen and M. B. van Gijzen: Dept. of Applied Math. Anal., Delft University of Technology, REPORT 09-02 (2009).

[5] M. Tanio and M. Sugihara: Mathematical Engineering Technical Reports, The University of Tokyo, METR2009-16 (2009).

[6] M. Nakashima, S. Fujino, K. Tateiba and Y. Onoue: IEICE Trans. Electron. J92-C [4] (2009) 111 (in Japanese).

[7] J. M. Song and W. C. Chew: Microw. Opt. Technol. Lett. 10 (1995) 14. DOI: $10.1002 /$ mop. 4650100107

[8] S. M. Rao, D. R. Wilton and A. W. Glisson: IEEE Trans. Antenn. Propag. AP-30 [3] (1982) 409. DOI:10.1109/TAP.1982.1142818

[9] Fast and Efficient Algorithms in Computational Electromagnetics, ed. W. C. Chew, J.-M. Jin, E. Michielssen and J. M. Song (Artech House Publishers, Boston-London, 2001). 


\section{Introduction}

In recent years, one of the Krylov subspace methods, i.e., the induced dimension reduction (IDR) method [1], has attracted considerable attention in the field of computational physics. The unique feature of the $\operatorname{IDR}(s)$ method is that the residual vector converges to a zero vector because the dimensions of the spaces to which the residual vector belongs decrease monotonically, in accordance with the IDR theorem. Mathematically, it requires at most $N+N / s$ matrix-vector multiplications (MATVEC) to obtain the exact solution, while conventional Bi-CG based methods need $2 \mathrm{~N}$ MATVEC.

Meanwhile, $\operatorname{IDR}(s)$ possesses an inherent problem, the so-called "spurious convergence". It is a phenomenon where the true relative residual norm (TRRN) at convergence becomes much larger than the value of the predefined tolerance. To avoid the problem, Sonneveld developed Bi-IDR $(s)$ in 2008 [2], in which bi-orthogonality is imposed on an auxiliary vector in the $\operatorname{IDR}(s)$ algorithm. By doing so, both the computational stability and computational complexity are improved [3]. Besides, $\operatorname{IDR}(s)$ has been developed in another way. By applying higher-order stabilization polynomials to the $\operatorname{IDR}(s)$, $\operatorname{IDR}(s) \operatorname{stab}(L)$ and $\operatorname{GIDR}(s, L)$ were derived by Sleijpen et al. [4] and Tanio et al. [5], respectively, in 2009. These two methods are algorithmically different, but share the same mathematical essentials. Recently, the performance of these IDR variants has been discussed in some papers such as $[3,6]$; however, thus far, it has hardly been studied or used for electromagnetic wave problems. Against this background, the main objective of the present study is to investigate the convergence performance and tolerance to the spurious convergence of the $\operatorname{IDR}$ variant methods, i.e., $\operatorname{IDR}(s), \operatorname{Bi}-\operatorname{IDR}(s)$, and $\operatorname{GIDR}(s, L)$, implemented along with the method of moments (MoM) for solving largescale and practical electromagnetic scattering problems.

This paper is organized as follows. In section 2, an overview of the essentials for the IDR variant methods is provided. In section 3, the results of numerical experiments are presented to discuss the performance of the IDR variant methods. Finally, in section 4, the paper is summarized and some concluding remarks are provided.

\section{IDR variant methods}

We consider a linear system $A \boldsymbol{x}=\boldsymbol{b}$, where $A \in C^{N \times N}$ represents a nonHermitian coefficient matrix; $\boldsymbol{x} \in C^{N}$, an unknown solution vector; and $\boldsymbol{b} \in C^{N}$, a right-hand side vector. Krylov subspace methods iteratively update the solution vector $\boldsymbol{x}_{k+1}$ such that the corresponding residual vector $\boldsymbol{c}$ belongs to the Krylov subspace $K_{k+2}\left(A, \boldsymbol{r}_{0}\right)$; the iterations continue until the predefined stopping criteria are satisfied. In this section, we sum up the essentials for the IDR variant methods.

\section{$\operatorname{IDR}(\mathbf{s})$}

First, we summarize the IDR theorem. 


\section{Theorem 1 IDR theorem}

Let $A$ be any matrix in $C^{N \times N}, \boldsymbol{v}_{0}$ be any vector in $C^{N}$, and $\boldsymbol{g}_{0}$ be the complete Krylov space $K_{N}\left(A, \boldsymbol{v}_{0}\right)$. Now, let $S$ denote any subspace of $C^{N}$. We define the sequence spaces $\boldsymbol{g}_{j}(j=1,2, \cdots)$ as

$$
g_{j}:=\left(I-\omega_{j} A\right)\left(g_{j-1} \cap S\right),
$$

where $\omega_{j}$ are non-zero scalars. Then, the following two theorems hold:

$$
\text { (i) } g_{j} \subseteq g_{j-1} \text { for all } j>0, \quad \text { (ii) } g_{j}=\{\mathbf{0}\} \text { for some } j \leq N
$$

Furthermore, without loss of generality, we may assume the space $S$ to be the left null space of some (full rank) $N \times s$ matrix $P$ :

$$
P=\left(\boldsymbol{p}_{1}, \boldsymbol{p}_{2}, \cdots, \boldsymbol{p}_{s}\right) . \quad S=N\left(P^{H}\right),
$$

where $\mathbf{N}$ represents the null-space and $H$ denotes the Hermitian adjoint.

In the $\operatorname{IDR}(s)$ method, $\boldsymbol{x}_{k+1}$ is determined such that $\boldsymbol{r}_{k+1}$ belongs to the subsequent space $g_{j+1}$ defined by Eq. (1). A notable feature of $\operatorname{IDR}(s)$ is that, in accordance with the IDR theorem, the residual vector $\boldsymbol{r}_{k+1}$ converges to a zero vector since the dimensions of the spaces $g_{j+1}$ decrease monotonically.

\section{$\operatorname{Bi}-\mathbf{I D R}(\mathbf{s})$}

In $\operatorname{Bi}-\operatorname{IDR}(s)$, the algorithm is designed such that the following two orthogonal conditions are satisfied:
(i) $\boldsymbol{g}_{n+i} \perp \boldsymbol{p}_{j}(i=2, \cdots, s, j=1, \cdots, i-1)$
(ii) $\boldsymbol{r}_{n+i+1} \perp \boldsymbol{p}_{j}(i=1, \cdots, s, j=1, \cdots, i)$

Here, $\boldsymbol{g}_{n+i}:=\boldsymbol{r}_{n+i}-\boldsymbol{r}_{n+i+1}$ and $\boldsymbol{p}_{j}$ indicates the column vector of $P$. By imposing the above two conditions, $\operatorname{Bi}-\operatorname{IDR}(s)$ achieves not only the computational stability, but also the reduction of computational complexity.

\section{$\operatorname{GIDR}(\mathbf{s}, \mathbf{L})$}

$\operatorname{GIDR}(s, L)$ is based on a generalized IDR theorem. While $\boldsymbol{g}_{j}$ of IDR theorem is constructed by multiplying 1-degree polynomial of $A$ to $g_{j}$, we now consider a space $g_{j}^{L}$, which is derived by generalizing the 1-degree polynomial to $L$ degree polynomial. For the implementation of these IDR variant methods, we referred to the algorithms described in [1] for $\operatorname{IDR}(s)$, [2] for $\operatorname{Bi}-\operatorname{IDR}(s)$, and [5] for $\operatorname{GIDR}(s, L)$, respectively.

\section{Numerical experiments}

In this section, we present numerical results and discuss the performance of the IDR variant methods. For the numerical experiments, we consider the following three objects made of perfectly electric conductor (PEC): (a) Sphere, (b) NASA almond, and (c) Airplane.

The first test case (a) is a scattering problem with a PEC sphere. The sphere has a radius of $16 \lambda$, and it is discretized into 672,996 triangular meshes, leading to 1,009,494 unknowns. The NASA almond is considered in the test case (b). The dimensions of the almond are $32.02 \lambda \times 12.35 \lambda \times 4.20 \lambda$; 206,646 meshes are generated, resulting in a total of 309,969 unknowns. The 
third test example (c) involves an aircraft with dimensions $26.07 \lambda \times 15.03 \lambda \times$ $3.74 \lambda$; this geometry is discretized into 73,599 triangular meshes, and the number of degrees of freedom of the resulting linear system is 110,293. In all of the above-mentioned test cases, the scatterers are illuminated by an $x$ polarized incident plane wave traveling along the $z$-axis. For the implementation of MoM, we employ the combined field integral equation (CFIE) [7], and the RWG basis functions [8] for surface current expansion. In addition, the Multilevel Fast Multipole Algorithm (MLFMA) [9] is incorporated to alleviate the computational cost of a MATVEC. We conduct comparative experiments with $\operatorname{IDR}(s), \operatorname{Bi}-\operatorname{IDR}(s), \operatorname{GIDR}(s, L)$, and $\operatorname{GMRES}(m)$. Here, GMRES $(m)$ represents the restarted version of GMRES. We use two stopping criteria: a tolerance of 1.0E-8 and a maximum iteration number of 5,000. Diagonal scaling preconditioner is applied to all the solvers. All the runs are performed in double precision on an Itanium 2 processor on an SGI Altix 450 server that has $128 \mathrm{~GB}$ memory.

Firstly, we investigate the spurious convergence problem that the $\operatorname{IDR}(s)$ algorithm inherently possesses. The spurious convergence is a phenomenon where the true relative residual norm (TRRN) computed by $\left\|A \boldsymbol{x}_{n}-\boldsymbol{b}\right\|_{2} /\|\boldsymbol{b}\|_{2}$ at the convergence becomes much larger than the value of the predefined tolerance, and this phenomenon tends to frequently occur when the parameter $s$ is set to large number. Fig. 1 depicts the TRRN versus the parameter $s$ for the three test cases. The geometry for each problem is also illustrated in the inset of each figure. The parameter $s$ ranges from 2 to 30 at intervals of 2 . For $\operatorname{GIDR}(s, L), L$ is selected as $1,2,4$, and 8 . First, it is worth noting that the characteristics of $\operatorname{IDR}(s)$ against the parameter $s$ strongly depend on the geometry of the problem; i.e., when the scatterer has a simple shape, the characteristics is almost independent of $s$, whereas if the geometry is complex, $\operatorname{IDR}(s)$ is vulnerable to the effects of the selection of $s$. As Nakashima stated in [6], since the spurious convergence stems from the singularity or ill-conditionedness of the $s \times s$ linear system related to the matrix $P$ in Eq. (2), it occurs frequently when $s$ is large. In addition, the numerical instability caused by the spurious convergence propagates and accumulates its influence as the number of iterations grows. Hence, we must set $s$ to an adequately small value to avoid the spurious convergence, for a problem with a complex geometry where we need a large number of iterations for convergence. In contrast, Bi$\operatorname{IDR}(s)$ appears to have quite robust characteristics for the spurious convergence, since the TRRN always stays around 1.0E-8 regardless of both the selection of $s$ and the geometry complexity. As for $\operatorname{GIDR}(s, L)$, it also occasionally encounters the numerical instability resulted from the spurious convergence when $s$ and $L$ are large. In Fig. 1, we see that the TRRN gradually increases and the method suffers from the spurious convergence, when the $s$ value exceeds approximately 20 .

Fig. 2 indicates the number of MATVEC required for the convergence as a function of the parameter $s$. Again, the inset of each figure illustrates the geometry of each problem. In the results for the test case (a) and (b), it cannot be seen any significant differences among $\operatorname{IDR}(s), \operatorname{Bi}-\operatorname{IDR}(s)$, and $\operatorname{GIDR}(s, L)$ 

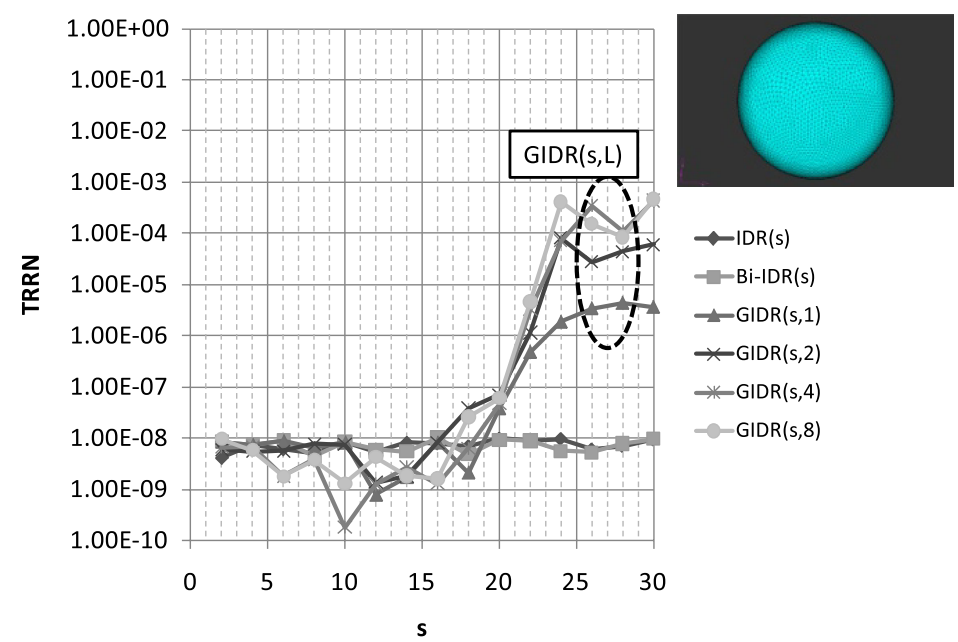

(a) Sphere

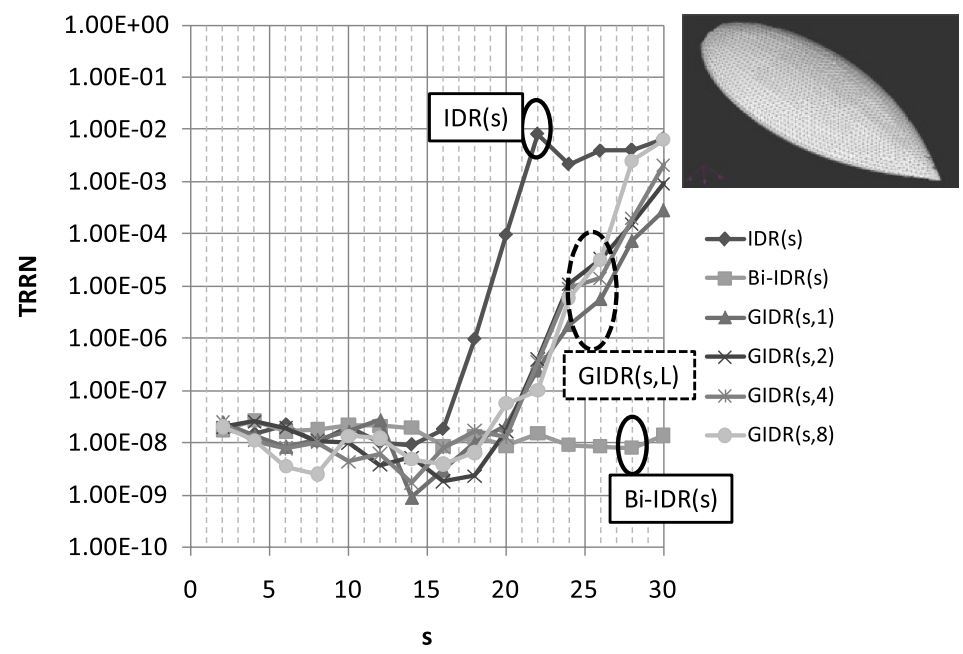

(b) NASA almond

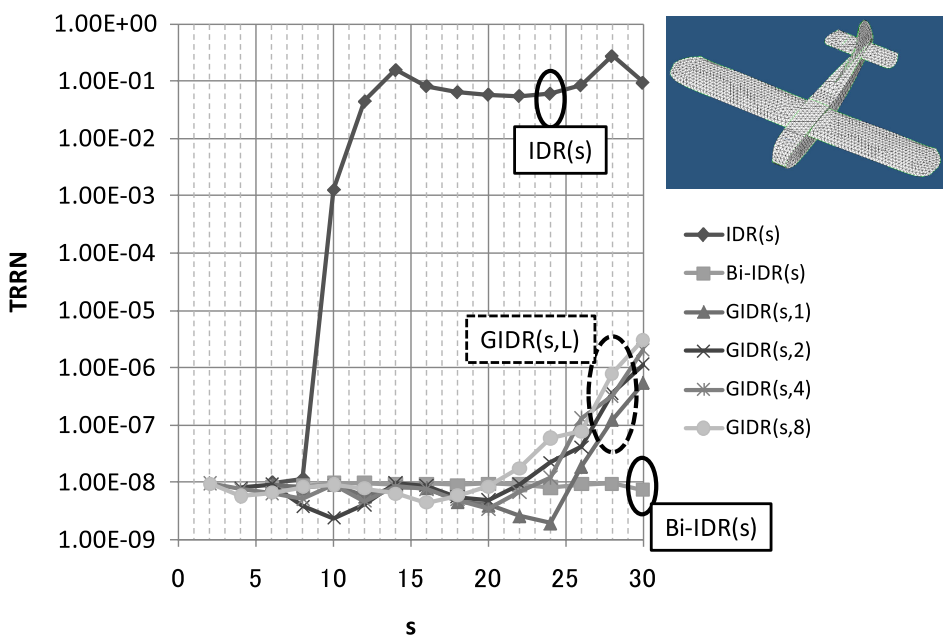

(c) Airplane

Fig. 1. TRRN as a function of the parameter $s$.

\section{IEICE 2014}

DOI: $10.1587 /$ elex.11.20140198

Received March 5, 2014 


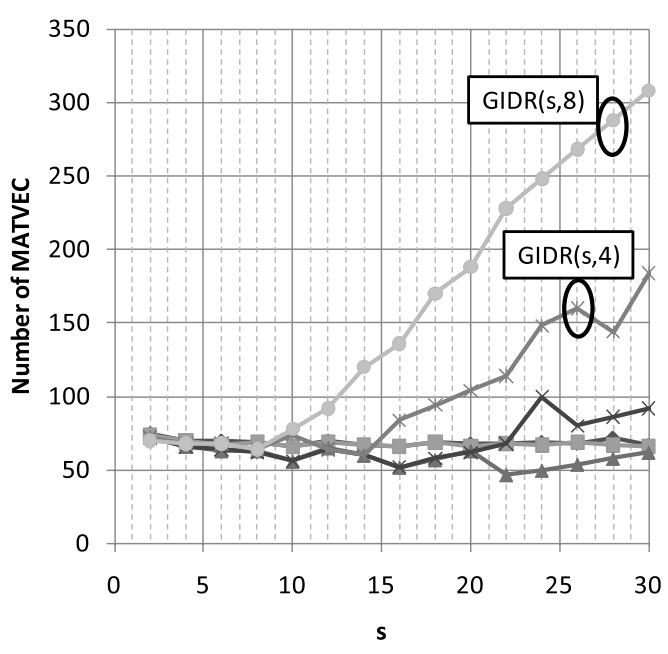

(a) Sphere

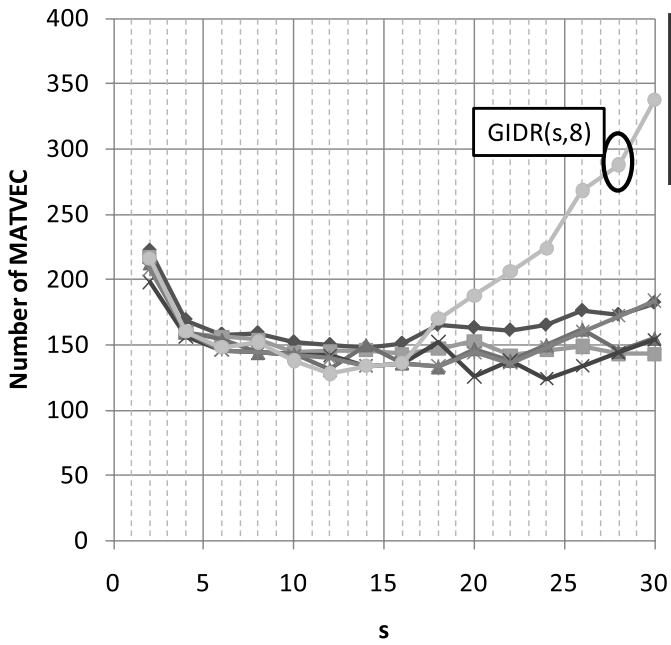

(b) NASA almond

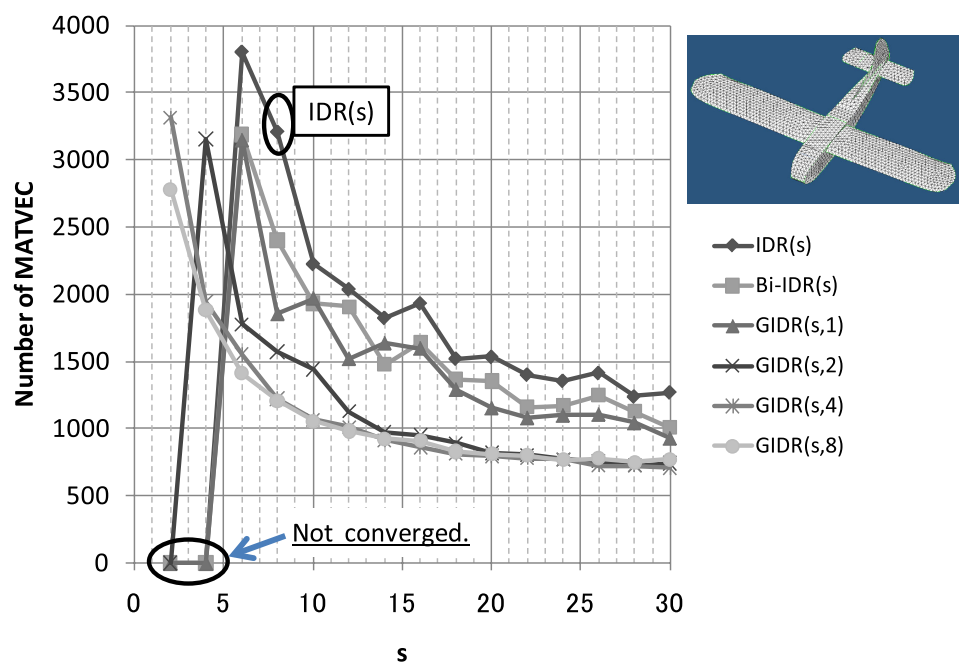

(c) Airplane

Fig. 2. Number of MATVEC against the parameter $s$. 
with a lower value of $L$, whereas $\operatorname{GIDR}(s, L)$ with a higher value of $L$ requires far larger numbers of MATVEC. This phenomenon is also due to the numerical instability of $\operatorname{GIDR}(s, L)$ with large $s$ and $L$. Particularly, in case where both $s$ and $L$ are set to large number, we infer that the numerical error tends to accumulate, and the numerical instability becomes distinctive. On the contrary, in the test case (c), we can find different tendencies; $\operatorname{GIDR}(s, L)$ with higher values of $L$, say, $L=4$ or 8 , always requires much less MATVEC than other methods. This is because, for this problem with a complex geometry, the convergence improvement obtained by the $\operatorname{GIDR}(s, L)$ appears more remarkably compared to the influence of the numerical instability. According to all the observations obtained from Fig. 1 and Fig. 2, the following facts have been clarified:

- When using $\operatorname{IDR}(s)$, one needs to carefully select the parameter $s$; especially, when the geometry of the problem is complex, $\operatorname{IDR}(s)$ is vulnerable to the effects of the parameter $s$ due to the spurious convergence problem. $s=8$ would be the appropriate selection for several levels of problem complexity.

- $\operatorname{Bi}-\operatorname{IDR}(s)$ never faces the spurious convergence problem regardless of both the selection of $s$ and the geometry complexity, whereas its convergence rate is inferior to that of $\operatorname{GIDR}(s, L)$.

- With an appropriate selection of $s$ and $L, \operatorname{GIDR}(s, L)$ shows a much faster convergence property than $\operatorname{IDR}(s)$ and $\operatorname{Bi}-\operatorname{IDR}(s)$ do. However, for large $s$, the method still suffers from the spurious convergence, and the convergence property appears to be distinctively deteriorated. From our numerical experiments, the selection of $L=2$ or 4 and an $s$ value of

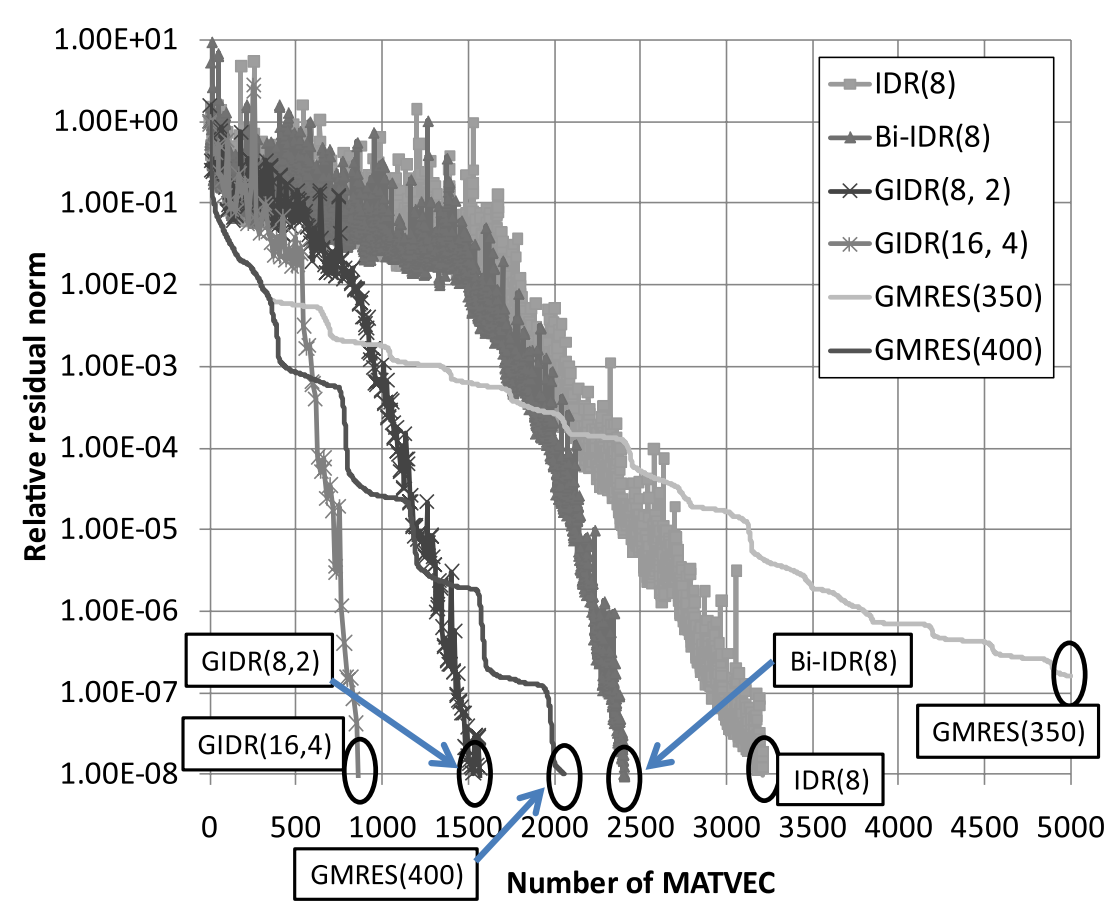

Fig. 3. Convergence histories of $\operatorname{IDR}(8), \operatorname{Bi}-\operatorname{IDR}(8)$, $\operatorname{GIDR}(8,2), \quad \operatorname{GIDR}(16,4), \quad \operatorname{GMRES}(350), \quad$ and GMRES(400) for the test case (c) with an airplane. 
Table I. Memory usage required for the convergence of $\operatorname{IDR}(s), \operatorname{Bi}-\operatorname{IDR}(s), \operatorname{GIDR}(s, L)$ and $\operatorname{GMRES}(m)$

\begin{tabular}{c|c|c|c}
\hline Iterative method & Sphere & NASA almond & Airplane \\
\hline $\operatorname{IDR}(8)$ & 11.97 & 4.40 & 3.78 \\
$\operatorname{Bi}-\operatorname{IDR}(8)$ & 11.81 & 4.35 & 3.76 \\
$\operatorname{GIDR}(8,2)$ & 12.05 & 4.42 & 3.79 \\
$\operatorname{GIDR}(16,4)$ & 13.11 & 4.74 & 3.90 \\
$\operatorname{GMRES}(m)$ & $12.51(m=50)$ & $4.56(m=50)$ & $4.38(m=350)$ \\
$\operatorname{GMRES}(m)$ & $13.38(m=100)$ & $4.80(m=100)$ & $4.44(m=400)$ \\
\hline
\end{tabular}

around 15 gives the best performance with respect to the balance between the convergence property and the tolerance to spurious convergence.

Fig. 3 compares the convergence histories for the test case (c) with an airplane. It is apparent that $\operatorname{GIDR}(16,4)$ converges much faster than other methods.

Finally, Table I lists the memory usage. It is noteworthy that, especially in the most complex problem (c), since $\operatorname{GMRES}(m)$ requires a quite large restart cycle to converge, it consumes high amounts of large memory. This result leads to the conclusion that IDR variants have an apparent advantage in terms of memory requirements over $\operatorname{GMRES}(m)$.

\section{Conclusion}

In this paper, we presented the performance of the IDR variant methods implemented with the MoM for solving electromagnetic scattering problems involving large conducting objects with complex geometries. Numerical experiments revealed that $\operatorname{GIDR}(s, L)$, when $s$ and $L$ are appropriately selected, showed a predominant convergence property as compared to $\operatorname{IDR}(s)$ and Bi$\operatorname{IDR}(s)$. We can conclude that the selection of $L=2$ or 4 and an s value of around 15 gives the best performance with respect to the balance between the convergence property and the tolerance to spurious convergence for problems with several levels of geometry complexity. 